\title{
Survey of Location Aware Mobi-Feed System
}

\author{
Patil Tejashree $\mathbf{R}^{1}$, Gaikwad Nivasini $\mathbf{A}^{2}$ \\ Student, Computer Science \& Engg, DACOE, Karad, India ${ }^{1,2}$
}

\begin{abstract}
An Location aware mobi-feed framework empowers portable clients to get every continuous way area data and the highlighted news. Here an office get gave to clients that he/she can pick any intrigued area. This paper shows a mobi-encourage framework which keeps versatile clients overhauled portable clients from new news of any spatial and non-spatial location. In this paper we have introduced three key modules that are, client module, server module, web seek module. This area mindful mobi-nourish framework separates itself from all current mobi-bolster frameworks that it empowers clients for getting different fundamental data of a specific area just utilizing this one application rather utilizing distinctive applications for various hunts furthermore clients can pick spatial area as opposed to static point area. The area mindful mobi-bolster framework keeps the portable clients overhauled from progressing or forthcoming areas and the imperative news of any area.
\end{abstract}

Keywords: Location Aware, mobi-feed, Location Aware News Feed.

\section{INTRODUCTION}

A mobi feed news maintain system engages versatile customers to share geo-marked customer created
messages, e.g., a customer can draw near by messages that are the most appropriate to her. In this paper, we show MobiFeed that is a framework expected for booking news feeds for versatile customers. MobiFeed includes three key limits; zone desire, hugeness measure, and news sustain scheduler. The region estimate limit is planned to anticipate a flexible customer's territories in light of a present way desire computation. The congruity measure limit is completed by joining the vector space exhibit with non-spatial and spatial factors to choose the significance of a message to a customer. The news maintains scheduler works with the other two abilities to deliver news reinforces for an adaptable customer at her present and foreseen territories with the best broad quality. To ensure that MobiFeed can scale up to a greater number of messages, we plot heuristic news manage scheduler.

A news support is a run of the mill handiness of existing territory careful casual association structures. It enables compact customers to post geo-marked messages and draw nearby customer made messages, e.g.," Alice can get 4 messages that are the most imperative to her among the messages inside $1 \mathrm{~km}$ from her zone at normal interims". Since a range careful interpersonal association system generally speaking has a colossal number of messages, there are many messages in a scrutinizing customer's district. Consolidated with customer movability, a key test for the zone careful news energizes system is the best approach to successfully arrange the $\mathrm{k}$ most critical messages for a customer and show them on the customer's phone.

Notwithstanding the way that territory careful news reinforce and interpersonal association systems have pulled in an extensive measure of thought from different research bunches, none of these applications has focused on the most capable technique to date-book news reinforces for compact customers. Instead of Geo-Feed, Mobi-Feed focuses on challenges in giving zone careful news supports to flexible customers. We diagram a region careful Mobi-Feed scheduler that works with our territory desire and message significance measure abilities to offer newsfeeds to versatile customers. In this paper, we show Mobi-Feed framework expected for casual group structures to schedule news feeds for versatile customers. Alice can in like manner issue Location careful Mobi-Feed request to recuperate the $\mathrm{k}$ most essential messages inside her predefined broaden isolate D from her region. MobiFeed contains three key limits: zone desire, congruity measure, and news support scheduler .Given a customer user's region u:location right now t0, user's required slightest message demonstrate time td, user's predefined broaden evacuate $\mathrm{D}$, user's requested number of messages per news feed, and a look-ahead steps $n$,the zone estimate work gages $\mathrm{n}$ future territories for $\mathrm{u}$ at timest $1=\mathrm{t} 0+\mathrm{td}, \mathrm{t} 2$ $=\mathrm{t} 0+2 \times \mathrm{td}, \ldots$, and $\mathrm{tn}=\mathrm{t} 0+\mathrm{n} \times \mathrm{td}$, the relevance measure work discovers the importance score of each candidate message with a geo-range meeting any user's question region and the news empower scheduler produces news supports from the contender messages for user's request regions at $\mathrm{t} 0, \mathrm{t} 1, \ldots .$. , tn with the best total essentialness score.

Arranging a flexible and convincing Mobi-Feed scheduler has a couple key troubles. A message has a lifetime concerning a customer's improvement. A message can be a cheerful message for a couple of consecutive or nonconsecutive news supports. The base show times of these news empowers constitute the message's lifetime. The scheduler should pick at most $\mathrm{k}$ candidate messages for a news energize inside their lifetime breaks to such a degree, to the point that the general way of a customer's news sustains is expanded. The relevance of a message to a customer is exceedingly evolving. Since we consider the detachment between a message and a customer as one of 
IARJSET

the factors in the relevance measure, the significance of a every one of the clients close-by and display it to the message could change for a customer at different collector.

territories. A customer needs to have the most pertinent 2) Classified Relevance Measuring- To furnish the client message at the most elevated purpose of a result list. The with various classifications to pick as his inclinations and congruity of a message appeared on a screen should be create message which are important to that specific class. weighted by its position. For example, the most raised 3) News Feed producing- Create a news nourish with weight is given to the message appeared at the top on the every one of the redesigns of different clients around that screen. The online scheduler should be viable with the true objective that it could scale up to innumerable.

\section{A. Background of project:}

A mobi-feed framework is the accumulation of different voyaging related information, that's why it will be beneficial for any versatile client which give present and up and coming areas data.

With the advances in situating systems, for example, GPS, cell-towers and Wi-Fi, clients can appreciate area based administrations more effectively than any time in recent memory, e.g., on their PDAs and GPS gadgets. Therefore, area based administrations have been exceptionally mainstream. The majority of the area based administrations can be dreamy as various sorts of spatial inquiries to a POI database or the street systems, for cases, 1) spatial range inquiry, which may locate the close-by eateries in a given spatial range from the clients, 2) kclosest neighbour question, which may discover the closest $\mathrm{k}$ service stations from the client's present area, 3) shortest way question, which can give the client with the most helpful course to her goal. In any case, with the ubiquity of proposal (e.g., Amazon \&Netflix) and socialization (like Facebook and Twitter) functionalities in the web benefits, the term Web 2.0 gets to be extremely famous, which is connected with web applications that encourage participatory data sharing, swarm sourcing, interoperability, client focused outline, and cooperation on the World Wide Web. The fame of Web 2.0 occurred as an immediate consequence of the wide increment of online client produced substance and long range informal communication innovations. In Web 2.0, the World Wide Web has moved from being an interface for data recovery to an intelligent medium where clients can share data, transfer client created content, and interface with different clients.

\section{B. Demand of Project:}

\section{- Advantages:}

1. Maximize the significance of the area based nourish while the bolster fulfilled the h-expanded requirement to differentiate the substance of the nourish.

2. To enhance the nature of the news nourish by not simply looking for the comparable substance but rather including all the client created redesigns from a differentiated arrangement of classifications.

- The application significantly utilizes 4 primary calculations to produce important yields and make a news nourish for the client:

1) Way Predicting- To decide the correct geo-area of the client in order to accumulate all the news overhauls from geo-area.

4) Enhancement checking- To create messages similarly from various picked classifications and making it broadened while being introduced to the client.

\section{Existing System:}

Existing LANF systems fundamentally send the most noteworthy geo-marked messages to their customers. Lamentably, the genuine imperative of such a present approach is, to the point that, a news manage may contain messages related to a comparable region (i.e., reason for interest) or a comparative class of ranges (e.g., sustenance, redirection or diversion). A news support is a common convenience of existing LBSNs. It enables adaptable customers to post geo-marked messages and get nearby customer created messages as news empowers at whatever time, wherever. The widening issues focus on recouping an individual once-over of things with a particular level of contrasting qualities.

\section{Proposed System:}

In this venture we propose a consider a portable situation that makes an area and assorted qualities mindful news encourage framework one of a kind and additionally difficult. With the present area, current time and classification (eq. Shopping centre, Hotel, Restaurant, Museum, Church) a message and Mobile client in significance measure display. We give a gcm warning to versatile client furthermore send notice to then again specific client. An area forecast procedure we go for enhancing the nature of news bolster by booking numerous area also, assorted qualities mindful news encourage framework for portable client at the same time. In this venture assorted qualities is critical highlight for location mindful news encourage on the grounds that it helps client find new place and exercises.

\section{RELATED WORK}

A) Geo-Feed: A Location-Aware News Feed System: A location aware news feed system that takes into account the spatial extents of messages and user locations when deciding upon the selected news feed.

Geo-Feed is equipped with three different approaches, namely spatial pull, spatial push and shared push for delivering the news feed to its users. Based on an accurate developed cost model for each approach, Geo-Feed employs a smart decision algorithm that decides about using these approaches in a way that: (a) minimizes the system overhead for delivering the location-aware news feed, and (b) guarantees a certain response time for each 
IARJSET

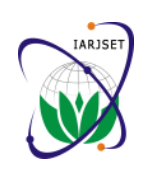

International Advanced Research Journal in Science, Engineering and Technology

National Conference on Innovative Applications and Research in Computer Science and Engineering (NCIARCSE-2017)

AGTI's Dr. Daulatrao Aher College Engineering, Vidyanagar Extension, Karad

Vol. 4, Special Issue 4, January 2017

user to obtain her location aware news feed. Geo-Feed investigation comes about demonstrate that Mobi-Feed further extends the spatial push approach to support the gives productive and brilliant news sustains for portable moving users. Experimental results, based on real and clients.

synthetic data, show that Geo-Feed is favourable over existing news feed systems, with a minimal system overhead.[1]

B) Mobi-Feed: A Location-Aware News Feed Framework for Moving Users: Mobi-Feed that is a framework designed for scheduling location-aware news feeds for mobile users. Mobi-Feed has three key functions: location prediction, relevance measure, and news feed scheduler. This proposed the n-look-ahead heuristic and optimal news feed schedulers that work with the locations. This evaluated other two functions to generate news feeds for a user at her current and $n$ future predicted the performance of Mobi-Feed through extensive experiments using a real road map, a real location-aware social network data set and a synthetic data set. The experiment results show that Mobi-Feed provides efficient and high-quality news feeds for mobile users.[2]

C) Location Service Based on News-feed: The D-MobiFeed location-aware news feed framework takes the relevance and diversity of news feeds into account when scheduling news feeds for moving user. It aims at maximizing the total relevance of generated newsfeed and satisfying the diversity constraint. The two key problems in D-Mobi-Feed, namely, decision and optimization problems. The decision problem is modelled as a maximum flow problem and enables D-Mobi-Feed to decide whether it can fulfil the diversity constraint for a news feed. Experimental results based on a real social network data set crawled from Foursquare and a real road network show that D-Mobi Feed can efficiently provide location- and diversity-aware news feeds when maintaining their high quality in terms of relevance. [3]

\section{CONCLUSION}

We displayed Mobi-Feed that is an area mindful mobisupport system intended for planning news sustains for portable clients. An area mindful mobi maintain structure framework foresee current and non spatial areas news or any related data for voyaging client. We portrayed the three key elements of Mobi-Feed, in particular, area forecast, importance measure, and news encourage scheduler. The area expectation capacity is intended to gauge a client's area in view of the way forecast calculation. The pertinence measure capacity is actualized by coordinating non-spatial and spatial variables into the vector space model to quantify the importance of a message to a user. The news bolster scheduler that works with the other two capacities to create news encourages for a client at her current and look-ahead areas. It minimizes the framework overhead to deliver the area mindful news bolster, and ensures a specific reaction time for every client to acquire her area mindful news encourage. The

[1] Jie Bao1,Mohamed F,Mokbel1,Chi-YinChow2. "Geo-Feed: A Location-Aware News Feed System".. Department of Computer Science and Engineering, University of Minnesota, Minneapolis, MN, USA.

[2] Wenjian $\mathrm{Xu} \cdot$ Chi-Yin Chow · Man Lung ,Yiu · Qing Li · Chung Keung Poon . "Mobi-Feed: A Location-Aware News Feed Framework for Moving Users".May,2015,IEEE Transactions on services computing.

[3] K.Prathiba1, A.Petrisia2 1PG Student, Associate Professor Department of MCA, Dhanalakshmi Srinivasan College of Engineering and Technology. "Location Service Based on Newsfeed" International Journal of Research in Engineering Technology -- Volume 1 Issue 4, May - June 2016.

[4] Siddharth Mishra,Toom Arjun Reddy,Pradeep Sadanand."A Location based diversified mobile news feed system(Android").March 2016,JETIR(ISSN-2349-5162)

[5] Jie Bao. "Towards Location-aware News Feeds and Recommendations". June 2014.

[6] J. Bao, M. F. Mokbel, and C.-Y. Chow. "Geo-Feed: A locationaware news feed system. "In IEEE ICDE, 2012.

\section{BIOGRAPHIES}

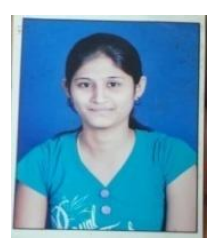

Tejashree R Patil. received Diploma in Computer Technology from CST Atit (Satara) College of Polytechnic in 2014. Appearing B. E. degree at of Dr. Daulatrao Aher College of Engineering, Karad, District Satara.

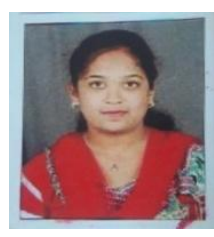

Nivasini A Gaikwad received Diploma in Information Technology from GRW polytechnic Tasgoan (Sangli) in 2014. Appearing B. E. degree at of Dr. Daulatrao Aher College of Engineering, Karad, District Satara. 